\title{
Large transconductance oscillations in a single-well vertical Aharonov-Bohm interferometer
}

\author{
Vincenzo Piazza, ${ }^{1}$ Fabio Beltram, ${ }^{1}$ Werner Wegscheider, ${ }^{2,3}$ Chi-Te Liang, ${ }^{4,5}$ and M. Pepper ${ }^{4}$ \\ ${ }^{1}$ Scuola Normale Superiore and Istituto Nazionale per la Fisica della Materia, I-56126 Pisa, Italy \\ ${ }^{2}$ Walter Schottky Institute, Munich, Germany \\ ${ }^{3}$ Universität Regensburg, Regensburg, Germany \\ ${ }^{4}$ Cavendish Laboratories, University of Cambridge, Cambridge CB3 OHE, United Kingdom \\ ${ }^{5}$ Department of Physics, National Taiwan University, Taipei 106, Taiwan
}

(Received 18 July 2000)

\begin{abstract}
Aharonov-Bohm $(\mathrm{AB})$ interference is reported for the first time in the conductance of a vertical nanostructure based on a single $\mathrm{GaAs} / \mathrm{Al}_{x} \mathrm{Ga}_{1-x} \mathrm{As}$ quantum well $(\mathrm{QW})$. The two lowest subbands of the well are spatially separated by the Hartree barrier originating from electronic repulsion in the modulation-doped QW and provide $\mathrm{AB}$ two-path geometry. Split-gates control the in-plane electronic momentum dispersion. In our system, we have clearly demonstrated $\mathrm{AB}$ interference in both electrostatic and magnetic modes. In the latter case the magnetic field was applied parallel to the QW plane, and perpendicular to the $0.02 \mu \mathrm{m}^{2} \mathrm{AB}$ loop. In the electrostatic mode of operation the single-QW scheme adopted led to large transconductance oscillations with relative amplitudes exceeding $30 \%$. The relevance of the present design strategy for the implementation of coherent nanoelectronic devices is underlined.
\end{abstract}

The pioneering work by Aharonov and Bohm had farreaching fundamental implications as it pointed out the significance of potentials with respect to forces in quantum mechanics. ${ }^{1}$ In their paper, Aharonov and Bohm demonstrated that electrons propagating in the two arms of a ringlike structure experience a relative phase shift of $e / \hbar \int \mathbf{A} \cdot d \boldsymbol{l}$ (where $\mathbf{A}$ is the magnetic vector potential and the integral is performed along the ring) when a magnetic flux encircled by the ring is applied. This is true even if the classical Lorentz force is exactly zero along the electron path. A similar effect was predicted if one of the two arms was subjected to a change of its electrostatic potential, while keeping the electric field at zero by means of Faraday cages. If electrons injected and collected into/from the structure by means of leads propagate coherently, the phase shift was shown to manifest macroscopically in observable quantities such as conductivity. The latter was predicted to oscillate following cycles of constructive and destructive electronic interference.

Since its proposal, Aharonov-Bohm (AB) interference was demonstrated in several mesoscopic systems. ${ }^{2-7}$ The unprecedented advancement in semiconductor growth and nanofabrication technologies has opened the way to device applications of this effect. Indeed semiconductor devices based on electrostatic $\mathrm{AB}$ effect were proposed as building blocks for the development of coherent nanoelectronics. ${ }^{8,9}$ The advantages offered by quantum-interference devices with respect to conventional "classical" ones are very significant: a quantum interference transistor requires much smaller gate voltages compared to conventional field-effect transistors (some millivolts compared to volts) and this is expected to lead to higher transconductance and extremely small power dissipation. ${ }^{10}$

The AB effect relies on electronic phase coherence, however, and its observation in semiconductor heterostructures with marked and useful conductance oscillations demands high-quality materials and very careful design. Datta et al. ${ }^{3}$ pioneered the field and demonstrated for the first time magnetic $\mathrm{AB}$ oscillation in a $\mathrm{GaAs} / \mathrm{Al}_{x} \mathrm{Ga}_{1-x} \mathrm{As}$ heterostructure with a contrast (defined as the ratio between the peak-to-peak amplitude and the mean value) smaller than $1 \%$. Since then significant progress was made in the quality of materials and in structure design, but even some more recent demonstrations of $\mathrm{AB}$ effect in semiconductor ring structures showed a contrast of the magnetic $\mathrm{AB}$ signal of only about $10 \%{ }^{11}$

In light of successful applications of the $A B$ effect to quantum devices, it is of crucial importance to achieve much higher contrasts and implement the electrostatic $\mathrm{AB}$ configuration. In this communication we propose and demonstrate a scheme for an $\mathrm{AB}$ interferometer based on a single modulation-doped wide GaAs quantum well. The two transport paths originate from the two lowest subbands of the well and take advantage of the $\sim \AA$ resolution achieved by epitaxial growth techniques. Spatial separation between the two electronic paths is given by the formation of a Hartree barrier in the well, lateral confinement is provided by surface gating.

The samples were fabricated from a high-mobility singlequantum-well GaAs/ $\mathrm{Al}_{x} \mathrm{Ga}_{1-x}$ As heterostructure containing a two-dimensional electron gas (2DEG) with a Hall mobility of $5 \times 10^{6} \mathrm{~cm}^{2} \mathrm{~V}^{-1} \mathrm{~s}^{-1}$ and a total carrier density of 5.8 $\times 10^{11} \mathrm{~cm}^{-2}$ at $4.2 \mathrm{~K}$ after illumination. Carriers were distributed in the two lowest subbands and Shubnikov-de Haas measurements yielded $3.9 \times 10^{11} \mathrm{~cm}^{-2}$ electrons in the ground subband and $1.7 \times 10^{11} \mathrm{~cm}^{-2}$ in the first-excited one. The $60-\mathrm{nm}$ GaAs quantum well is located $75 \mathrm{~nm}$ below the surface and is embedded between $\mathrm{Al}_{0.25} \mathrm{Ga}_{0.75} \mathrm{As}$ barriers. Two $\delta$-doping layers are placed $35 \mathrm{~nm}$ (surface side) and 60 $\mathrm{nm}$ (substrate side) from the well. The choice of a wide single well instead of a double-quantum-well structure was motivated by the twofold need for spatially separated transport paths and long coherence lengths. In this scheme, spatial separation is guaranteed by the Hartree barrier formed by Coulomb repulsion between carriers populating the modulation-doped quantum well (see inset in Fig. 1). At the same time, the absence of a central $\mathrm{Al}_{x} \mathrm{Ga}_{1-x}$ As barrier reduces interface roughness scattering and alloy scattering.

One-dimensional leads are necessary to avoid smearing of the interference pattern in the electrostatic $\mathrm{AB}$ effect. ${ }^{8}$ Indeed, while for the magnetic $A B$ effect phase shifts depend 


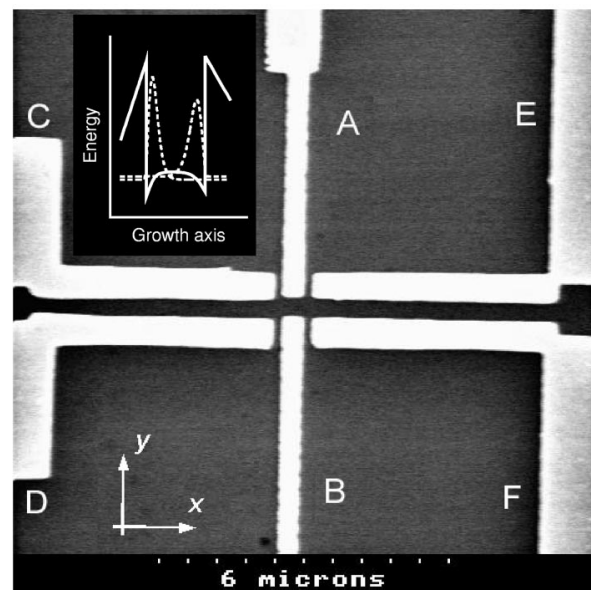

FIG. 1. Scanning electron micrograph of one of the devices studied. Cr-Au nanogates are shown as bright regions and define a set of wires in the $x$ direction. The Aharonov-Bohm ring is formed in the plane perpendicular to that of the image. Path separation arises from the Hartree barrier formed by Coulomb repulsion between carriers populating the modulation-doped quantum well. The inset shows the calculated conduction-band diagram (solid line) and the two lowest subband wave functions (dashed lines) of the quantum well at equilibrium at $4.2 \mathrm{~K}$.

on the area enclosed by the two paths, for the electrostatic $\mathrm{AB}$ effect the shift depends on the transit time of electrons in the two arms of the ring. ${ }^{8}$ Consequently injection of an electron beam with no in-plane momentum dispersion is required for $\mathrm{AB}$ observation. To this end, an injector and a collector were realized on a Hall bar by patterning two long $(5 \mu \mathrm{m})$ split gates on the surface of the sample. (C, D and E, F in Fig. 1). The geometrical width of the gap between the electrodes is $0.5 \mu \mathrm{m}$.) By appropriately biasing these gates it is possible to tune the number of modes propagating in the leads and, in the limit of zero temperature, in-plane momentum dispersion will be suppressed when a single mode is available for transport.

Given an estimated coherence length of about $1 \mu \mathrm{m}$, electronic transport in the leads is expected to be diffusive. This is required in our scheme to avoid coherent reflections at their ends that could hinder the observation of the main interference effect. The injector and collector were individually characterized by measuring the low-temperature conductance of the sample versus the voltage $V_{\mathrm{CD}}\left(V_{\mathrm{EF}}\right)$ applied to the split gate $\mathrm{C}, \mathrm{D}(\mathrm{E}, \mathrm{F}){ }^{12}$ None of them displayed clear signatures of conductance quantization, confirming the diffusive nature of electron transport.

A third split gate (A, B) was used to define the ballistic one-dimensional region and tune the phase difference between propagating paths. Electrons can be reflected back or transmitted to the collector region depending on the interference being destructive or constructive. The split-gate length was chosen to be $0.6 \mu \mathrm{m}$, shorter than the estimated coherence length. Indeed the conductance of the device versus the bias $\left(V_{\mathrm{AB}}\right)$ of the quantum point contact (QPC) defined by $A$ and $B$ displayed clearly quantized steps.

Figure 2 shows the conductance of the device at different values of $V_{\mathrm{AB}}$ as a function of $V_{\mathrm{CDEF}}=V_{\mathrm{CD}}=V_{\mathrm{EF}}$. Sweeping $V_{\mathrm{CDEF}}$ from zero bias toward negative values, a onedimensional channel is created as the 2DEG below the gates

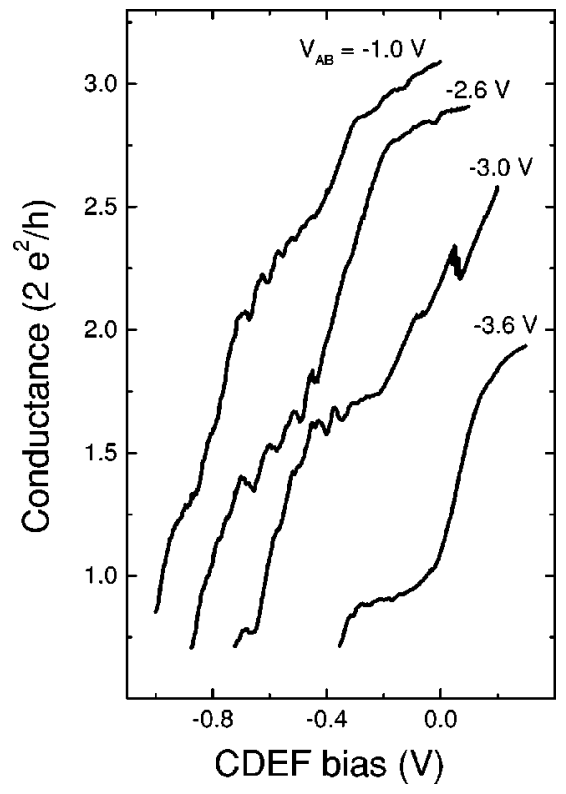

FIG. 2. Differential conductance measurements at $300 \mathrm{mK}$ as a function of the bias applied to gates C, D, E, and F (see Fig. 1). Four curves are plotted for different biases applied to gates $\mathrm{A}$ and B.

is depleted and then progressively narrowed until channel pinchoff at $\approx-1 \mathrm{~V}$. The value of the conductance in units of $2 e^{2} / h$ at a fixed $V_{\mathrm{AB}}$ and at $V_{\mathrm{CDEF}}=0$ can be read as the number of propagating modes in the QPC. For $-3 \mathrm{~V}<V_{\mathrm{AB}}$ $<-1 \mathrm{~V}$, corresponding to two to more than three modes in the QPC, very clear oscillations can be observed over a monotonically decreasing background as $V_{\mathrm{CDEF}}$ is swept below $\approx-0.5 \mathrm{~V}$. No oscillations could be seen when less than two modes are propagating in the QPC $\left(V_{\mathrm{AB}}\right.$ $<-3 \mathrm{~V}$ ), that is when only a single path is available for transport.

Figure 3 reports a set of oscillations obtained on one such

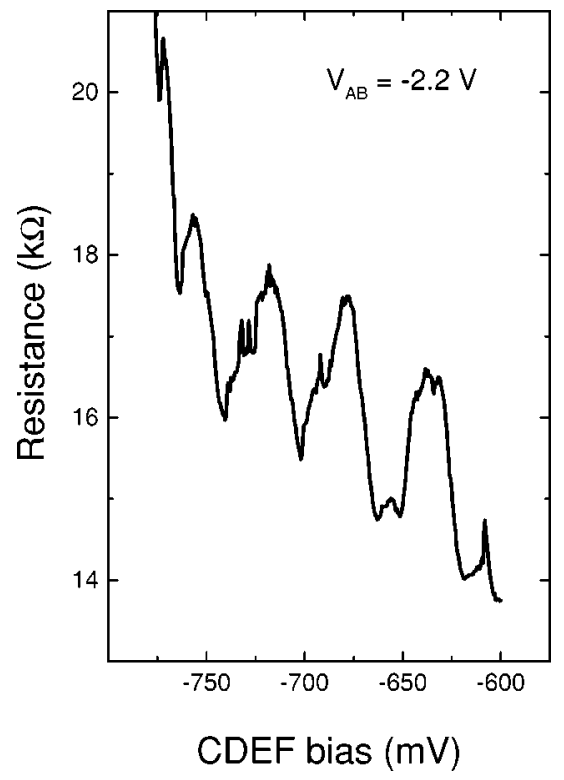

FIG. 3. Differential resistance at $300 \mathrm{mK}$ as a function of the bias applied to gates C, D, E, and F. Gates A and B were biased at $-2.2 \mathrm{~V}$. 
device. Subtracting the contribution to the resistance due to the one-dimensional diffusive leads $(\sim 10 \mathrm{k} \Omega)$ the contrast of the oscillations exceeds $30 \%$. To our knowledge, this value is the highest reported in the literature for semiconductor-heterostructure electrostatic $\mathrm{AB}$ rings and makes the present fabrication strategy of interest for device applications. Indeed the present structure does implement a possible quantum-interference transistor that yields a normalized transconductance $d I_{D} / d V_{G} \times 1 / I_{D} \gtrsim 35 \mathrm{~V}^{-1}$.

In order to understand the nature of the states (paths) involved in the interference processes it is necessary to distinguish between different situations. For sake of simplicity, we focus on the case of two propagating modes in the QPC. This configuration can occur in two different ways: (i) only the first two transverse modes originating from the lower quantum-well subband are below the Fermi level and all modes originating from the upper subband are depleted; (ii) electrons occupy the first transverse mode of each one of the two quantum-well subbands. To understand which of these possibilities is of interest here, we measured the conductance of our device at appropriately chosen gate biases as a function of an in-plane magnetic field perpendicular to the current. The magnetic field will couple to the loop formed by the two propagating paths and lead to magnetic AB oscillations only when configuration (ii) is realized. As reported in Fig. 4, very clear conductance oscillations can be seen as a function of magnetic field. Consistently with the results reported above, interference effects occur only when $V_{\mathrm{CDEF}}$ is swept below $\approx-0.5 \mathrm{~V}$. Again, no oscillations can be resolved when a single mode is accessible in the QPC. The period of $\mathrm{AB}$ oscillations is given by $\Delta B=h / e S$, where $S$ $=L d \approx 0.02 \mu \mathrm{m}^{2}$ is the area enclosed by the two paths, $d$ is the center-to-center distance between the channels and $L$ is the length of the two channels. For our sample we estimate $L \approx 0.6 \mu \mathrm{m}$ from the geometrical width of the QPC and $d$ $\approx 35 \mathrm{~nm}$, obtained from a self-consistent calculation of the two subband wave functions. These values give a theoretical period $\Delta \mathrm{B} \approx 0.19 \mathrm{~T}$, in good agreement with the values of 0.2 $\mathrm{T}$ and $0.26 \mathrm{~T}$ measured from the data with $V_{\mathrm{CDEF}}=-0.5 \mathrm{~V}$ and $V_{\mathrm{CDEF}}=-1.2 \mathrm{~V}$, respectively (in both cases $V_{\mathrm{AB}}$ $=-2.5 \mathrm{~V}$ ). The greater period measured in the latter case is linked to the increased wave function confinement and consequent reduction of $\mathrm{AB}$ loop area.

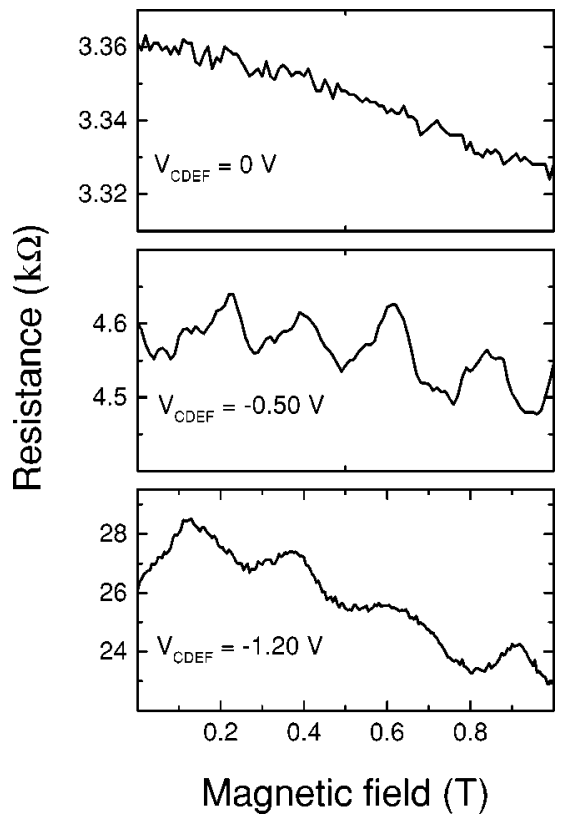

FIG. 4. Differential conductance at $300 \mathrm{mK}$ as a function of the external magnetic field applied in the $y$ direction (see Fig. 1). The three panels show data for different biases applied to gates C, D, E, and F. Gates A and B were biased at $-2.5 \mathrm{~V}$.

In summary, we have demonstrated a scheme leading to very high-contrast Aharonov-Bohm interference driven both by an electric and a magnetic field in a high-mobility heterostructure. The analysis of the results allowed to unambiguously identify the states involved in the process. The present structure does implement a quantum interference transistor device that in its present configuration leads to a normalized transconductance analogous to that of classical devices.

This work was funded in part by the British Council and CRUI within the British-Italian collaboration scheme. The work at Cambridge was supported by the UK EPSRC. C.T.L. is grateful for the support from the NSC, Taiwan. Molecular beam epitaxial growth at the Walter Schottky Institut (TU Munchen) is supported by the Deutsche Forschungsgemeinschaft via Contract No. SFB 348 and the Bundesministerium für Bildung und Forschung under Contract No. 01 BM 630/1. We would like to thank Spiros Gardelis for his help in sample preparation.
${ }^{1}$ Y. Aharonov and D. Bohm, Phys. Rev. 115, 485 (1959).

${ }^{2}$ D. Yu. Sharvin and Yu. V. Sharvin, Zh. Éksp. Teor. Fiz. 34, 285 (1981) [JETP Lett. 34, 272 (1982)].

${ }^{3}$ S. Datta, M. R. Melloch, S. Bandyopadhyay, R. Noren, M. Vaziri, M. Miller, and R. Reifenberger, Phys. Rev. Lett. 55, 2344 (1985).

${ }^{4}$ G. Timp, A. M. Chang, J. E. Cunningham, T. Y. Chang, P. Mankiewich, R. Behringer, and R. E. Howard, Phys. Rev. Lett. 58, 2814 (1987).

${ }^{5}$ C. J. B. Ford, T. J. Thornton, R. Newbury, M. Pepper, H. Ahmed, J. T. Foxon, J. J. Harris, and C. Roberts, J. Phys. C 21, L325 (1988).

${ }^{6}$ D. A. Wharam, M. Pepper, R. Newbury, H. Ahmed, D. G. Hasko, D. C. Peacock, J. E. F. Frost, D. A. Ritchie, and G. A. C. Jones,
J. Phys.: Condens. Matter 21, 3369 (1989).

${ }^{7}$ M. Okuda, Appl. Phys. Lett. 63, 3309 (1993).

${ }^{8}$ S. Datta, M. R. Melloch, S. Bandyopadhyay, and M. S. Lundstrom, Appl. Phys. Lett. 48, 487 (1986).

${ }^{9}$ M. Okuda, K. Fujii, and A. Shimizu, Appl. Phys. Lett. 57, 2231 (1990).

${ }^{10}$ S. Datta, in Physics of Quantum Electron Devices, edited by F. Capasso (Springer-Verlag, Berlin, 1990).

${ }^{11}$ J. Appenzeller, Th. Schäpers, H. Hardtdegen, B. Lengeler, and H. Lüth, Phys. Rev. B 51, 4336 (1995).

${ }^{12}$ All the measurements were performed after illumination at 300 $\mathrm{mK}$ in a ${ }^{3} \mathrm{He}$ cryostat by four-terminal lock-in techniques, with a $10 \mathrm{nA}$ excitation current at $13 \mathrm{~Hz}$. 